\title{
Tasks of a feather flock together: Similarity effects in task switching
}

\author{
CATHERINE M. ARRINGTON \\ Vanderbilt University, Nashville, Tennessee \\ and \\ ERIK M. ALTMANN and THOMAS H. CARR \\ Michigan State University, East Lansing, Michigan
}

\begin{abstract}
Recent research on task switching has paid little attention to how tasks are represented and how the relations between task representations might affect the executive processes engaged to achieve a task switch. Two experiments investigated the effect of task similarity on task switching. Similarity was defined in terms of shared component operations-attentional control settings in Experiment 1 and response modality in Experiment 2-with tasks sharing more component operations said to be more similar to each other than tasks sharing fewer component operations. Across both experiments, task similarity facilitated task switching, seen in reduced switch costs for switching between similar tasks as opposed to dissimilar tasks. These results indicate that task similarity defined in terms of component operations can be used to define a multidimensional task space in which the executive processes of task selection and implementation are active.
\end{abstract}

Human cognition is noteworthy in its ability to deal with changes in environments and behavioral goals with apparent ease and flexibility. This ability can be seen in situations that demand sequential performance of two or more tasks, each of which may place very different demands on the perceptual, cognitive, and response systems. Selection and performance of a single task in the face of competing behavioral possibilities is thought to involve a combination of executive control and automatic processes (Logan, 1985; Norman \& Shallice, 1986; Posner \& Snyder, 1975). There has been a recent flurry of interest in studying these processes with the use of the task switching paradigm (Jersild, 1927).

\section{Task Switching}

The task switching paradigm involves simple tasks interleaved. Performance of these tasks is disrupted when a switch from one task to another is required. This disruption is evident when the performance of a given task $A$ on a trial that follows the performance of a different task B is contrasted with the performance of task A when

This work was supported in part by Grant SBR 0218507 from the National Science Foundation. These experiments were previously presented at the 43rd Annual Meeting of the Psychonomic Society in Kansas City, MO in 2002. This research represents a portion of the doctoral dissertation of the first author, who thanks her coauthors and the other members of her dissertation committee, Rose Zacks and Rick DeShon, for their guidance throughout this project. We thank Daniel Bouk and Emily Lauher for their help with data collection and Gordon Logan, Herbert Heuer, and Nachshon Meiran for helpful comments on this article. Correspondence concerning this article should be addressed to C. M. Arrington, Department of Psychology, 11121 st Ave. S., Nashville, TN 37203 (e-mail: kate.arrington @ vanderbilt.edu). it follows another trial of task A. The task switch condition $(\mathrm{B}-\mathrm{A})$ results in slower and less accurate performance than does the task repetition or no switch condition (A-A). The difference between these two conditions is referred to as switch cost. Explanations of switch cost are varied; it has been attributed to executive control processes that must be engaged to select and prepare the new task (Rogers \& Monsell, 1995), as well as to automatic influences on performing the new task due to residual activation in memory from recent performance of a different task (Allport, Styles, \& Hsieh, 1994). More recent accounts of switch cost have proposed combinations of executive control and automatic effects (Logan \& Gordon, 2001; Meiran, Chorev, \& Sapir, 2000; Sohn \& Anderson, 2001). All of these explanations focus on processes that occur when the cognitive system must be reconfigured between tasks. Other models of task switching explain switch cost in terms of broader memory phenomena (Altmann \& Gray, 2002; Logan \& Bundesen, 2003). Thus, there is considerable theoretical variety in this active and fluid research area.

Methodological variations and choices of tasks are also numerous. Many tasks have been used, including categorization of numbers (Logan \& Bundesen, 2003; Sudevan \& Taylor, 1987), letters (Rogers \& Monsell, 1995), or symbols (Arbuthnott \& Woodward, 2002); identification of colors or words using Stroop stimuli (Allport et al., 1994); location judgments (Meiran, 1996, 2000a); semantic and episodic memory tasks (Mayr \& Kliegl, 2000); and arithmetic problems (Jersild, 1927; Rubinstein, Meyer, \& Evans, 2001). Typically both the stimuli and responses for these tasks are multivalent, in such a way that the same sets of stimuli and responses afford several tasks in 
which the stimulus-response (S-R) mappings are overlapping. Multivalent as opposed to univalent stimulus and response sets can result in interference or crosstalk among tasks, which may make switch costs more likely to occur (Meiran, 2000b; Rogers \& Monsell, 1995). Because the tasks are interleaved and the multivalent nature of the stimuli does not uniquely specify the task to be performed, the appropriate task on any given trial must be specified in some manner. Researchers have developed a number of methods for indicating the current task, including presenting tasks in a pre-set or alternating order (i.e., AABBAABB; Rogers \& Monsell, 1995); linking a feature of the target display to the identity of the task, such as location or color of the stimulus (Sohn \& Anderson, 2001); or providing instructional cues on each trial (Meiran, 1996) or prior to a run of trials (Altmann \& Gray, 2002; Gopher, 1996).

As might be suggested by this variety, the focus of task switching experiments is on the processes engaged when tasks are changed, rather than on the performance of the individual tasks. The standard calculation of switch cost, the subtraction of task repetitions from task switches, represents an attempt to isolate processes associated with changing between tasks while subtracting out the time associated with performing the tasks themselves. This view of task switching suggests the potential importance that relations among tasks might have on the processes engaged when one must shift from one task to another. Nevertheless, research on task switching has largely ignored the question of how various tasks are related to each other, as well as the effect of these relations on task transitions.

There have been two exceptions to this disregard for task relations. The first consists of work on the asymmetrical switch costs that arise when tasks are differentially dominant (Allport et al., 1994; Meuter \& Allport, 1999; Monsell, Yeung, \& Azuma, 2000). Dominance is a relative concept: one task is more practiced and more automatic than the other. As Monsell et al. have demonstrated, this concept can be difficult to specify for particular pairs of tasks. The second exception consists of work examining the number of components that change between one task and another. Studies examining whether task switches involve changes in one or two components of the tasks have provided conflicting results as to whether or not this variable affects task switching, with early evidence suggesting no effect (Allport et al., 1994), but more recent studies showing some conditions under which increased switch costs arise when two task components must be switched (Hübner, Futterer, \& Steinhauser, 2001).

Kleinsorge and colleagues have taken an additional step, examining both the number and also the nature of task components that differ between two tasks (Kleinsorge \& Heuer, 1999; Kleinsorge, Heuer, \& Schmidtke, 2001; see also Meiran \& Marciano, 2002). This work, which has asked whether it matters that tasks differ in a component whose services are needed earlier or later in the tasks' performance timeline, begins to consider the content of the tasks-what particular processes they engage and what stimulus and response representations they operate on.

In the present research, we develop this approach further. We are motivated by the idea that to understand the processes involved in switching between two tasks, it would be useful to systematically characterize, compare, and manipulate the task components on which these processes act. That is, task components must themselves be mentally represented, and the processes that choose between tasks and implement one task rather than another must operate on these mental representations of task components. Thus, we are beginning to ask how tasks are represented in the mind, and more specifically, what aspects of their representations are important to task switching.

\section{Task Space and Task Similarity}

The representation of a group of tasks may be conceptualized as a task space (Kleinsorge, 2000). Two factors can be used to define task space: the task sets that represent specific tasks, and the relations among these task sets. ${ }^{1} \mathrm{~A}$ task set can generally be thought of as a group of component cognitive processes or operations involved in performance of a task, with each of these components requiring a particular type of input representation, operating on the representation in some particular way, and producing a particular type of output representation that is communicated to other components in the sequence of processes that constitutes the task's real-time performance. A task set includes three major classes of components: perception or encoding of the stimulus, manipulations of or judgments about the stimulus, and response selection, programming, and execution. The representations and processes of the components involved in performing the task being switched from determine what needs to be abandoned during a task switch; the representations and processes involved in performing the task being switched to determine what needs to be activated during a task switch. The second factor defining task spacethe relations between task sets-can be described in terms of the placement of the task set in a multidimensional space in which these classes of components of task sets form the dimensions, and particular components within each class form the values along the dimensions. The specific cognitive operations involved in performing a particular task determine the task set's placement in task space.

The relations among task sets in task space can be characterized in terms of the similarity between pairs of tasks. When task sets are described in terms of the cognitive operations that are necessary for achieving the task's goals, then task similarity can be defined as the extent to which task sets share the specific component operations within each class. For any given class of component operations, task sets are more similar when they share the operation, and less similar when they do not (cf. Tversky, 1977). For example, all else being equal, if the perceptual encoding components and their operating characteristics are the same in two tasks, the two tasks are more similar 
than if the perceptual encoding components and their operating characteristics are different. If the response components and their operating characteristics are the same, the two tasks are more similar than if the response components and their operating characteristics are different. Note that although we define task similarity in terms of shared task components, the construct of similarity in cognitive psychology is controversial and has been defined in various ways (for a review see, e.g., Medin, Goldstone, \& Gentner, 1993). Thus, other measures of similarity might be applied to tasks and task sets. However, with the relative scarcity of research on the representations of tasks, it is not clear that one approach to defining similarity would be suggested over another at this point. As will be seen, our choice of shared task components proves to have heuristic value in beginning to address the question of how relations among task representations affect task switching.

Similarity between tasks is theoretically significant because, on the basis of existing research, one could predict either that similarity will facilitate task switching or that it will impair task switching. On the one hand, if task sets are viewed as points in a multidimensional similarity space, task switching may be thought of as a process of moving from one point to another, in line with reconfiguration models of task switching (Rogers \& Monsell, 1995). On this view, similar task sets would be closer together, and switching between them would be easier because the distance to be traversed is smaller. Thus, it may be easier to switch between similar tasks than between dissimilar tasks, because similar tasks have more component processes in common, so that fewer of them have to be changed.

On the other hand, a long history within the dual-task literature suggests that similarity between tasks might cause interference, not facilitation (McLeod, 1977; but see Heuer, 1996, for a discussion of circumstances that might give rise to facilitation). In reviewing this literature, Pashler (1998) observes that "there is little doubt that task similarity can exacerbate interference" (p. 294). The increase in interference resulting from increasing similarity would predict slower switches between tasks that are similar. This idea is made explicit in Pashler's review of similarity in dual-task environments: "Similarity may be a key determinant of performance whenever people switch back and forth between tasks" (p. 295).

However, there is a major difference between the kinds of dual-task situations reviewed by Pashler (1998) and the kinds of situations commonly examined in studies of task switching. This difference consists in the time delay between the onsets of the stimuli for successive tasks. Pashler was concerned primarily with the "psychological refractory period" (PRP) paradigm, in which stimuli requiring separate responses occur close together in time, often so close that the second stimulus occurs before the response to the first stimulus has been executed. Thus, performance of the two tasks overlaps in time, and the concern is with discriminating component operations that can be carried out in parallel from component operations that must be conducted one at a time. Tasks do not overlap in task switching paradigms, and the concern is with what must be done to change to a different task, given that one has already completed performance of the immediately preceding task. Thus, it is an empirical question at this point whether similarity will facilitate or interfere with sequential task performance once overlap between the two tasks has been removed, and the answer will have important theoretical consequences for understanding the mental representations of tasks and the control processes that operate on them.

We conducted two experiments to determine whether task similarity facilitates or impairs task switching. In the first experiment, we manipulated similarity of perceptual encoding operations, in particular of attentional control settings that govern which stimulus characteristics the visual attentional system will be most sensitive to (Folk, Remington, \& Johnston, 1992). In the second experiment, we manipulated similarity in terms of motor output modality, an aspect of the component operations of response selection, programming, and execution. Both experiments involved four two-choice discrimination tasks. In each case, the four tasks formed two pairs of tasks in which the tasks were more similar within a pair, but less similar across pairs. Tasks were presented in an explicit task cuing paradigm in which the tasks occurred in a random order and participants were cued on each trial as to which task to perform. Thus, within the same block of trials, participants performed task repetitions, task switches from similar tasks, and task switches from dissimilar tasks. Effects of task similarity were assessed by comparing switch costs associated with switches from similar and dissimilar tasks.

\section{EXPERIMENT 1}

The four tasks involved judgments of the height, width, hue, or brightness of a rectangular target. Judgments of height and width both involve processing aspects of the spatial extent of the target; judgments of hue and brightness both involve processing surface properties of the target. Thus, performance of each pair of tasks demands attention to different aspects of the target stimulus. On the basis of Garner's (1974) theory of selective attention, the pairs of tasks - height and width, and hue and brightness-would be integral within a pair of tasks and separable between the pairs. The definition of task similarity within this context then becomes tasks that share a common component-attentional selection, in which the same attentional operation that selects relevant stimulus information for one of the tasks also selects the relevant stimulus information for the other task. This task component may be thought of as an attentional control setting (Folk et al., 1992; Logan \& Gordon, 2001). For example, height and width dimensions may both be considered to involve an attentional control setting for form, whereas hue and brightness may both be considered to involve an attentional control setting for color. Switching between 
height and width or between hue and brightness does not require a change in attentional control setting, but switching between height and hue, or width and brightness, does require a change in attentional control setting. The question at stake in Experiment 1 was whether sharing versus needing to change the attentional control setting matters when one is switching from one task to another.

\section{Method}

Participants. Experiment 1 included 20 participants from undergraduate psychology courses who participated in partial fulfillment of course requirements. All reported normal or corrected-to-normal visual acuity and color vision.

Apparatus and Stimuli. All stimuli were displayed and responses collected on a Dell Dimension computer running E-Prime software (Psychology Software Tools, 2000). The cues were the words HEIGHT, WIDTH, COLOR, and BRIGHT in black on a white background. The targets were 16 colored rectangles that varied along the four task dimensions. The rectangles were four different shapes formed by crossing two heights ( 32 or 48 pixels) and two widths (64 or 96 pixels). Each rectangle appeared in four different hue/brightness combinations: light green (in the Microsoft Office drawing palette, RGB 8525585 ), light blue (90 90 254), dark green (0 120 0), and dark blue (0 0 170).

Procedure. The trials began with a cue positioned just above the center of the display, which indicated what task to perform on that trial. Following a 500-msec delay, the target appeared on the screen below the cue. Both cue and target remained on the screen until a response was made, at which point the screen was cleared and remained blank for $100 \mathrm{msec}$. The responses were made on the home keys of a standard keyboard (asdfjkl;). Responses for each task were mapped to corresponding fingers on the left and right hands (e.g., for the height task, responses were made with left and right index fingers; for the width task, responses were made with the left and right middle fingers; etc.), resulting in unique responses for each task. Task-to-finger mappings were counterbalanced across participants.

Participants practiced single task blocks of 16 trials for each task, followed by 2 mixed task blocks of 128 trials. Data collection then occurred over 12 blocks of 128 trials. The participants were given accuracy and average response time (RT) feedback following each block and were instructed to work as quickly and accurately as possible.

\section{Results and Discussion}

Trials were sorted into 16 conditions based on the task performed on trial $n$ and trial $n-1$. The participants' mean RTs were calculated following the removal of error trials, the two trials following an error, and trials in which the RT was less than $200 \mathrm{msec}$ or greater than two standard deviations above the mean RT calculated for each condition for each participant. This procedure resulted in removal of $12.4 \%$ of the trials, leaving on average 84 trials per condition. Table 1 shows the mean RT and accuracy data for all 16 conditions, with performance on a given task presented in columns as a function of the task performed on the previous trial, presented in rows.

To assess the effect of task similarity on switching between tasks, switch costs were calculated by subtracting RT on task repetition trials from RT on task switch trials. Figure 1 shows mean switch costs separated by similarity and the identity of the task performed on trial $n$. Across all four tasks, switch costs were smaller for switches from similar tasks $(M=263 \mathrm{msec})$ than for switches from dis-
Table 1

Experiment 1: Means and Standard Errors for Response Times (in Milliseconds) and Accuracies for Trial Conditions Based on Task on Trial $n$ and Trial $n-1$

\begin{tabular}{|c|c|c|c|c|c|c|c|c|}
\hline \multirow[b]{3}{*}{ Trial $n-1$} & \multicolumn{8}{|c|}{ Trial $n$} \\
\hline & \multicolumn{2}{|c|}{ Height } & \multicolumn{2}{|c|}{ Width } & \multicolumn{2}{|c|}{ Color } & \multicolumn{2}{|c|}{ Bright } \\
\hline & $M$ & $S E$ & $M$ & $S E$ & $M$ & $S E$ & $M$ & $S E$ \\
\hline \multicolumn{9}{|c|}{ Response Times } \\
\hline Height & 615 & 22 & 796 & 50 & 818 & 50 & 980 & 65 \\
\hline Width & 882 & 45 & 569 & 19 & 857 & 51 & 946 & 56 \\
\hline Color & 933 & 52 & 883 & 61 & 547 & 20 & 913 & 48 \\
\hline Bright & 962 & 56 & 882 & 64 & 794 & 40 & 601 & 16 \\
\hline \multicolumn{9}{|c|}{ Accuracies } \\
\hline Height & 98.1 & 0.5 & 96.2 & 0.7 & 96.2 & 0.7 & 96.5 & 0.7 \\
\hline Width & 96.7 & 0.7 & 98.3 & 0.5 & 96.5 & 0.6 & 96.2 & 0.7 \\
\hline Color & 96.4 & 0.9 & 96.4 & 0.8 & 98.1 & 0.4 & 96.6 & 0.6 \\
\hline Bright & 96.5 & 0.7 & 96.4 & 0.7 & 96.9 & 0.4 & 97.1 & 0.6 \\
\hline
\end{tabular}

similar tasks $(M=324 \mathrm{msec})$, indicating a facilitation of task switching between similar tasks. Switch costs were entered into a 2 (similarity: similar or dissimilar) $\times 4$ (task: height, width, color, or bright) repeated measures analysis of variance (ANOVA). The analysis revealed that the 61-msec main effect of similarity was significant $\left[F(1,19)=15.6, M S_{\mathrm{e}}=9,619.3, p<.01\right]$. There was no effect of task $\left[F(3,57)=2.0, M S_{\mathrm{e}}=20,622.8, p=.12\right]$, and no interaction of the similarity effect with task $[F(3,57)=$ $\left.1.2, M S_{\mathrm{e}}=2,972.5, p=.32\right]$. Accuracy was highest on task repetitions $(M=97.9 \%)$ followed by switches from similar tasks $(M=96.6 \%)$ and switches from dissimilar tasks $(M=96.4 \%)$. A 2 (similarity) $\times 4$ (task) ANOVA on the accuracy data showed no significant effects. Thus, there was no evidence for a speed-accuracy tradeoff.

These results indicate a facilitation of task switching when tasks are similar. The pattern of faster responding following a switch from a similar task as opposed to a dissimilar task held true for all four tasks. The fact that the similarity effect did not interact with task indicates that the facilitation cannot be attributed to specific tasks that are more or less difficult to switch to or away from than other tasks (Allport et al., 1994; Monsell et al., 2000). Rather, the similarity effect results from the particular relations between pairs of tasks, which in this case have been defined in terms of shared attentional control settings. These results support the conclusion that the relative position of two task sets in task space defined in terms of the component operations shared by those tasks does indeed influence the processes engaged when switching between tasks. However, in Experiment 1, we tested only one kind of component operation (by manipulating attentional control settings, an aspect of perceptual encoding). To determine whether the benefit of task similarity generalizes to additional component operations, we conducted a second experiment in which we manipulated the nature of the component at the other end of the task's real-time performance-namely, control over response output modality. 


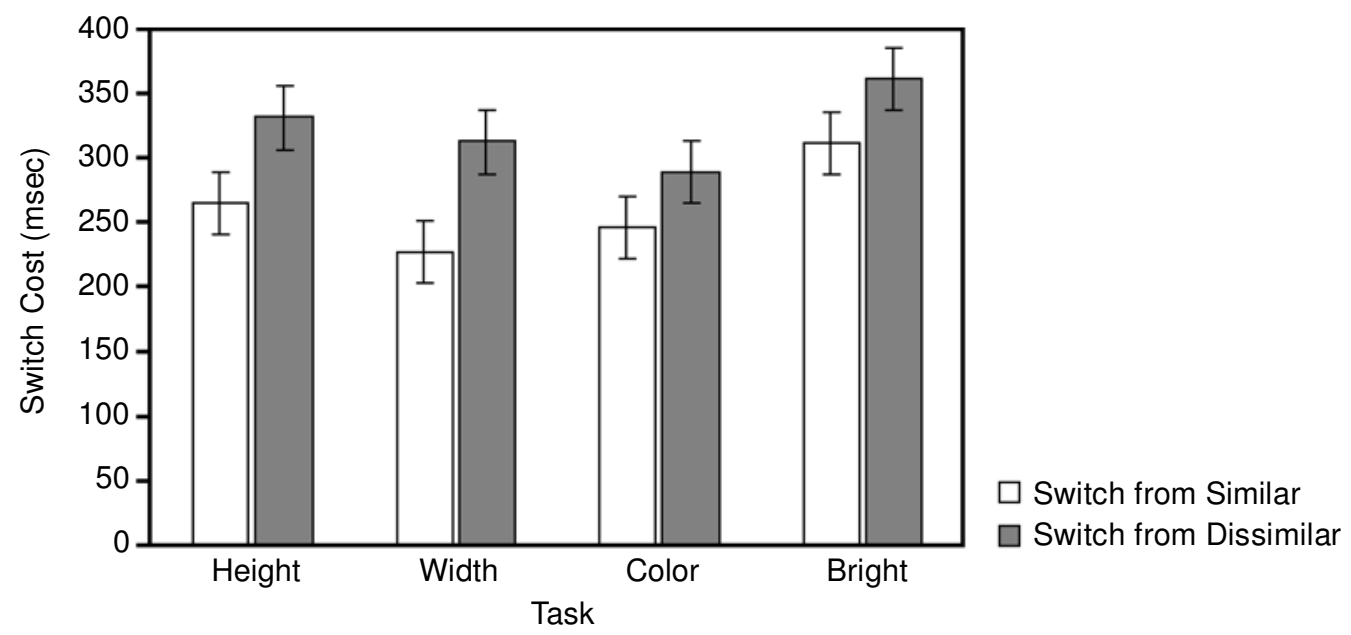

Figure 1. Experiment 1: Mean switch costs for switching from similar and dissimilar tasks for each task type. Error bars represent $95 \%$ confidence intervals calculated on the basis of the error term for the interaction of similarity $\times$ task as described in Loftus and Masson (1994).

\section{EXPERIMENT 2}

Experiment 2 introduced four new tasks that formed two pairs of similar tasks, with similarity defined in terms of response modality rather than attentional control setting. In each task, participants judged whether a rectangle was tall or short and indicated that judgment with a response that varied with task cue. Two tasks involved manual responses: one in which the participant used the first finger of the right and left hands to indicate target height; one in which the participant used the second finger of each hand. The other two tasks involved vocal responses: one in which the participants responded by saying a number, either "one" or "two," where "one" might indicate a tall rectangle and "two" might indicate a short rectangle; one in which the participant responded by saying a letter, either "A" or "B." The vocal tasks were developed so that the responses matched the manual responses in terms of being arbitrarily mapped to the stimulus values (i.e., using words such as "tall" or "short" would have provided a prepotent response, thus separating these responses from the manual responses in more ways than just modality).

Given the findings from Experiment 1 and the model of task space in which task similarity facilitates task switching, switches should be more rapid between tasks that share a response modality than between tasks that involve different response modalities. However, research examining response modality effects in other dual-task environments such as the PRP paradigm has shown greater interference in performance of the second task when two tasks are performed in the same response modality (manual-manual) as opposed to different response modalities (manual-vocal), although the PRP occurs in both instances (Pashler, 1990, 1998). This research suggests that similarity in the present set of tasks should impair task switching in such a way that switches should be slower between tasks that share a response modality than between tasks that involve different response modalities. As discussed earlier, it is an empirical question whether conclusions drawn from the PRP paradigm, in which tasks overlap in time, will hold for task switching, where one task is completed before the stimulus for the next task is presented.

\section{Method}

Except where noted, the method was the same as in Experiment 1. Twenty participants were drawn from the same population as in Experiment 1, with the additional restriction that they be native English speakers.

The instructional cues were FIRST, SECOND, NUMBER, and LETTER, indicating the type of response to be made, as described. The two target stimuli were the wide, light blue rectangles from Experiment 1 , varying only in terms of height. Responses were recorded with hardware from Psychology Software Tools (2000). Manual responses were made on four buttons on a button box. Vocal RTs were recorded with a voice key, and the identities of vocal responses were recorded by the experimenter, who remained in the experimental room. Additional audio recordings of the sessions were made to cross check accuracy of the vocal responses. During the instruction and practice period, the participants were also given instructions about appropriate use of the voice key. The participants then completed 10 blocks of 128 trials for data collection.

\section{Results and Discussion}

The general approach and procedures for data analysis were the same as in Experiment 1. In addition to the previous trimming procedures, trials on which there was a voice key error were removed. This procedure resulted in removal of $12.0 \%$ of the data, leaving on average 70 trials per condition. Table 2 shows the mean RTs and accuracies for the 16 conditions generated on the basis of task on trial $n$ and trial $n-1$.

The results of the switch cost data are consistent with those found in Experiment 1. Figure 2 shows the switch costs for switches from similar and dissimilar tasks sep- 
arated by task performed on trial $n$. Switch costs were smaller when the task transition involved a switch from a similar task $(M=173 \mathrm{msec})$ rather than a switch from a dissimilar task $(M=231 \mathrm{msec})$. The magnitudes of the switch costs are somewhat smaller than those for the comparable conditions in Experiment 1, possibly because the attended stimulus dimensions and the decision were the same for each of these four tasks, representing a greater number of shared component operations in this particular set of four tasks. However, the magnitude of the similarity effect $(58 \mathrm{msec})$ was similar to that observed in Experiment 1 . This main effect of task similarity was significant $\left[F(1,19)=15.4, M S_{\mathrm{e}}=8,640.1, p<.01\right]$. There was no effect of task $\left[F(3,57)=0.5, M S_{\mathrm{e}}=16,062.1, p=.69\right]$, and the similarity effect did not interact with task $[F(3,57)=$ $\left.0.8, M S_{\mathrm{e}}=4,036.6, p=.51\right]$. Accuracy was highest for task repetitions $(M=98.5 \%)$ followed by switches from similar tasks $(M=97.5 \%)$ and switches from dissimilar tasks $(M=97.3 \%)$. A 2 (similarity) $\times 4$ (task) ANOVA on the accuracy data showed no significant effects. This pattern of data suggests no speed-accuracy tradeoff.

The key finding in this experiment was the striking correspondence of the results to those from Experiment 1. The redefinition of task similarity in terms of response modality did not alter the pattern of the similarity effect. Once again, across all four tasks, switches from similar tasks occurred more rapidly than switches from dissimilar tasks. Taken together with the results from Experiment 1, these findings point to an effect of task similarity on the processes involved in switching from one task to another, where task similarity is defined in terms of shared component mental operations required for task performance.

\section{GENERAL DISCUSSION}

Across two experiments in which task similarity was defined as shared attentional control settings (Experiment 1 ) or shared response modality (Experiment 2), task similarity facilitated task switching. This facilitation occurred for all of the tasks tested in both experi-

Table 2

Experiment 2: Means and Standard Errors for Response Times (in Milliseconds) and Accuracies for Trial Conditions Based on Task on Trial $n$ and Trial $n-1$

\begin{tabular}{|c|c|c|c|c|c|c|c|c|}
\hline \multirow[b]{3}{*}{ Trial $n-1$} & \multicolumn{8}{|c|}{ Trial $n$} \\
\hline & \multicolumn{2}{|c|}{ First } & \multicolumn{2}{|c|}{ Second } & \multicolumn{2}{|c|}{ Number } & \multicolumn{2}{|c|}{ Letter } \\
\hline & $M$ & $S E$ & $M$ & $S E$ & $M$ & $S E$ & $M$ & $S E$ \\
\hline \multicolumn{9}{|c|}{ Response Times } \\
\hline First & 551 & 20 & 764 & 42 & 847 & 38 & 829 & 44 \\
\hline Second & 728 & 45 & 580 & 29 & 873 & 48 & 838 & 45 \\
\hline Number & 764 & 68 & 828 & 55 & 622 & 17 & 777 & 37 \\
\hline Letter & 759 & 68 & 847 & 53 & 793 & 41 & 617 & 16 \\
\hline \multicolumn{9}{|c|}{ Accuracies } \\
\hline First & 98.5 & 0.4 & 97.6 & 0.4 & 96.5 & 0.7 & 97.4 & 0.5 \\
\hline Second & 97.2 & 0.4 & 97.6 & 0.5 & 96.0 & 0.7 & 97.6 & 0.5 \\
\hline Number & 97.2 & 0.5 & 97.9 & 0.4 & 98.9 & 0.3 & 97.8 & 0.4 \\
\hline Letter & 98.1 & 0.4 & 97.8 & 0.5 & 97.4 & 0.4 & 98.9 & 0.3 \\
\hline
\end{tabular}

ments, ruling out the possibility that the results arose from idiosyncratic task-specific effects. Thus, these findings support the hypothesis that task switching is influenced by similarity within a task space defined by component cognitive operations. Similar tasks - those that share component operations-will be closer in task space than dissimilar tasks, thus facilitating a switch between them. We note that in both experiments, the magnitude of the similarity effect was substantially smaller than the overall switch costs. This finding fits well in the shared component operations account of the similarity effect that we are proposing. In terms of the degree of overlap of task components, task repetitions have substantially more overlap than do either switches from similar tasks or switches from dissimilar tasks.

Our view of task space complements that of Kleinsorge (2000; Kleinsorge \& Heuer, 1999; Kleinsorge et al., 2001), although our emphasis on the possible organization of task space differs. Kleinsorge's experiments defined a task environment in which four potential tasks were formed through an orthogonal manipulation of the type of judgment to be made on a stimulus, either spatial or numerical, and the response mapping, either compatible or incompatible. They referred to this set up as a dimensionally organized task space and focused on the hierarchical nature of the relations among tasks: Task judgment is superordinate to task mapping, which is in turn superordinate to specific responses made with either the left or the right hand. In their model of task space, a critical feature is the hierarchical organization, based on the temporal ordering of task dimensions-judgment, judgment-to-response mapping, and response dimensions are ordered on the basis of when in the process of performing a task they occur, with earlier occurring dimensions influencing the processes that act on later occurring dimensions. Our conceptualization of task space based on component operations is not inconsistent with this view, but it also allows for factors other than temporal order to play a critical role in organizing task space. Indeed, it would be interesting to examine the similarity effect within the context of a Kleinsorge-like temporal hierarchy, using tasks that combined both attentional and response modality manipulations of similarity. It is becoming quite clear that the relations among task sets are important to our understanding of how switching is achieved, and the more comprehensively we can characterize task space, the more precisely we will be able to characterize movements within it.

Drawing on theories of task switching, one can hypothesize two potential mechanisms for the task similarity effect. On the basis of theories of task switching that emphasize the executive control processes involved in active reconfiguration of task set in preparation for the upcoming task (Meiran, 2000a; Rogers \& Monsell, 1995; Rubinstein et al., 2001), the task similarity effect may arise from a reduction in the amount of preparation that must be carried out for switching between similar tasks as compared with dissimilar tasks. This decrease in preparation may be 


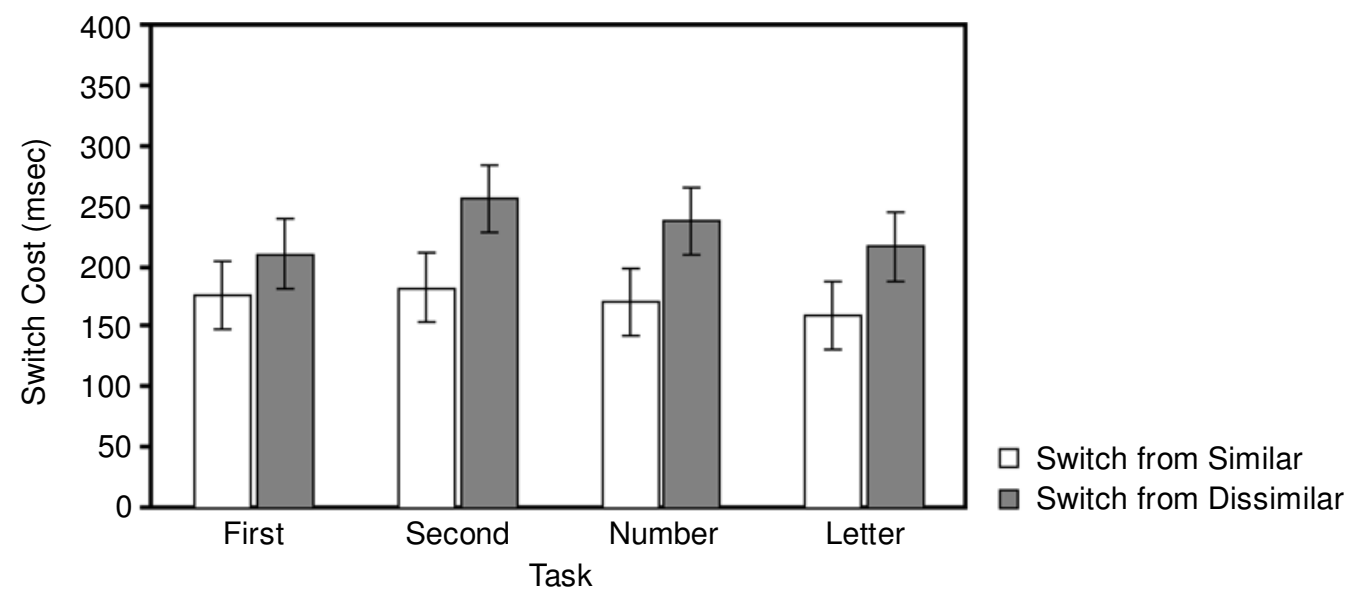

Figure 2. Experiment 2: Mean switch costs for switching from similar and dissimilar tasks for each task type. Error bars represent $95 \%$ confidence intervals calculated as in Experiment 1.

thought of in terms of a decrease in the distance between two task sets in the task space view that we have proposed, or as a decrease in the number of parameters that must be reset to prepare for the upcoming task (Logan \& Gordon, 2001).

Alternatively, the task similarity effect may arise from passive or automatic influences that act when tasks are performed closely in time. Several models of task switching evoke such automatic mechanisms, but differ in their details. Some propose interference arising from residual activation from the previous task (Allport et al., 1994) or from stimulus-triggered retrieval of previous S-R mappings (Allport \& Wylie, 1999, 2000). Others propose repetition priming, in which aspects of a task are performed more rapidly on task repetitions because they are primed from the previous trial (Sohn \& Anderson, 2001; Sohn \& Carlson, 2000). Similar tasks with more shared task components may prove to be more or less susceptible to such automatic influences based on the overlapping task sets. For example, in the case of a switch from a similar task, residual activation associated with the task set for the trial $n-1$ task would include some activation of the trial $n$ task set in which task components are shared. The result of the residual activation would thus be to boost the starting level of activation for the current task set, and the result would be priming for the portion of the task set that overlapped with the task set activated on the previous trial.

Ongoing studies in our laboratory, in which we have manipulated the preparation interval between cue and target and the delay interval between successive trials, indicate that both executive control and automatic processes influence the task similarity effect (Arrington, 2002). In addition, we have replicated the task similarity effect from Experiment 1, using an alternating-runs procedure (Rogers \& Monsell, 1995) in which task sequences take the form AABBCCDD, such that participants must generate and remember what task is to be performed next. The finding of task similarity effects in this context is important, because recent research has questioned whether explicit task-cuing procedures like those used here involve endogenous acts of task switching (Logan \& Bundesen, 2003).

The present experiments raise a question concerning boundaries for the task similarity effect: As tasks become increasingly dissimilar, will the cost of switching continue to increase? The answer is clearly no. Beginning with the work of Jersild (1927) and continuing recently with various other task switching methodologies (Meiran, 2000b; Rogers \& Monsell, 1995; Spector \& Biederman, 1976), studies have demonstrated that if stimuli are univalent (i.e., unique for each task to be performed), switch costs can be greatly reduced or even eliminated. In terms of similarity, however, tasks with unique stimulus sets would appear to be less similar than tasks with overlapping stimulus sets, suggesting a boundary for the task similarity effect. Thus, when the stimulus itself supplies the cue for what task to perform, the constraints on task switching may be altered in a fundamental way.

Also, increasing similarity may not always result in decreasing switch costs. Consider the results from Experiment 2: Switching between manual responses made with different fingers is faster than switching between a vocal and a manual response. One might extrapolate to a situation involving even more similar motor responses, in which the same set of finger responses is used for multiple tasks (bivalent responses rather than the univalent responses used in the present experiments) and predict even more rapid responding during switching between these tasks because of the increased similarity of the responses. However, studies have demonstrated that bivalent responses lead to greater switch costs than univalent responses (Meiran, 2000b), thus showing the opposite effect of that predicted on the basis of task similarity. Both of these examples involve changes in the degree to which the $\mathrm{S}-\mathrm{R}$ mappings overlap and, in particular, whether $\mathrm{S}-\mathrm{R}$ 
mappings are many-to-one, many-to-many, or one-tomany. Such variation in the structure of the S-R mapping may prove to be a critical factor in establishing boundaries of the task similarity effect. Although the task similarity effect that we have demonstrated in this study shows a systematic influence on task switching, clearly other task variables determine the exact nature of the processes engaged in task switching. Understanding how the task similarity effect interacts with these other variables remains to be discovered in future research.

As we argued earlier, results from the PRP literature (e.g., Pashler, 1990, 1998) suggest that task similarity might interfere with, rather than facilitate, the process of switching between tasks. The contrast with the present results reveals how one might compare task switching and PRP paradigms in the context of our task space framework. Broadly speaking, the task switching paradigm and the PRP paradigm both purport to study executive functioning in dual-task environments and indeed involve very similar methodologies (Pashler, 2000). Both paradigms involve performance of (at least) two discrete tasks in close temporal proximity. However, in the task switching paradigm, trials involving the performance of a single task occur in a sequential fashion, with no temporal overlap in the component operations (stimulus encoding, cognitive judgment, and response selection) involved in the performance of separate tasks. In contrast, in the PRP paradigm, two tasks are presented within a single trial. The time interval between presentation of the stimuli for each task - the stimulus onset asynchrony, or SOAis manipulated as a variable of interest. For the short SOAs typical for PRP studies, the stimuli associated with both tasks are presented prior to either response, thus allowing the component processes associated with each task to overlap temporally as well.

This temporal overlap in the task performance may be critical for understanding why similarity has seemingly opposite effects in PRP and task switching paradigms. When tasks are being performed simultaneously, crosstalk between these tasks, measured in terms of a compatibility effect of the stimuli or responses for each task, can result in interference between tasks. Logan and Schulkind (2000) demonstrated that crosstalk occurs when the same task (e.g., a magnitude judgment) is performed on both stimuli, but that crosstalk is alleviated when tasks are different (e.g., a magnitude judgment on one stimulus and a parity judgment on the other), suggesting that task similarity may indeed be a mediating factor in the occurrence of interference due to crosstalk between tasks. Thus, although sequential switching from one task to another is facilitated when two tasks share components (as our results show), simultaneousperformance of tasks that share components may be inhibited due to interference between the two tasks.

This interpretation maps to standard conceptions of executive control that distinguish between subordinate task-specific processes and the superordinate executive processes that coordinate them (see, e.g., Logan \& Gor- don, 2001). When a given task-specific process is required by two tasks concurrently, the executive must nonetheless sequence them, causing interference; however, when that process is required by two tasks sequentially, the executive runs faster when that process is already activated from the previous trial. Further examination of the effects of task similarity in concurrent and sequential processing modes, and understanding how the effects map to everyday multitasking, will be important issues to address in future research.

\section{REFERENCES}

Al 1 port , A., St yl es, E. A., \& Hsieh, S. (1994). Shifting intentional set: Exploring the dynamic control of tasks. In C. Umiltà \& M. Moscovitch (Eds.), Attention and performance XV: Conscious and nonconscious information processing (pp. 421-452). Cambridge: MA: MIT Press.

Al 1 por t, A., \& Wyl ie, G. (1999). Task-switching: Positive and negative priming of task-set. In G. W. Humphreys, J. Duncan, \& A. [M.] Treisman (Eds.), Attention, space, and action: Studies in cognitive neuroscience (pp. 273-296). Oxford: Oxford University Press.

Al 1 port , A., \& Wyl ie, G. (2000). Task switching, stimulus-response bindings, and negative priming. In S. Monsell \& J. Driver (Eds.), Attention and performance XVIII: Control of cognitive processes (pp. 35-70). Cambridge, MA: MIT Press.

Al t mann, E. M., \& Gray, W. D. (2002). Forgetting to remember: The functional relationship of decay and interference. Psychological Science, 13, 27-33.

Ar but hn ot t, K. D., \& Woodwar d, T. S. (2002). The influence of cuetask association and location on switch cost and alternating-switch cost. Canadian Journal of Experimental Psychology, 56, 18-29.

Ar $\mathrm{r}$ in gt on, C. M. (2002). Explorations in task space: Similarity effects on task switching. Unpublished doctoral dissertation, Michigan State University.

Fol k, C. L., Remin gt on, R. W., \& John st on, J. C. (1992). Involuntary covert orienting is contingent on attentional control settings. Journal of Experimental Psychology: Human Perception \& Performance, 18, 1030-1044.

Garner, W. R. (1974). The processing of information and structure. Potomac, MD: Erlbaum.

Gopher, D. (1996). Attention control: Explorations of the work of an executive controller. Cognitive Brain Research, 5, 23-38.

Heuer, H. (1996). Dual-task performance. In O. Neumann \& A. F. Sanders (Eds.), Handbookof perception and action: Vol. 4. Attention (pp. 35-70). London: Academic Press.

Hübner, R., Fut t er er, T., \& St ein h au ser, M. (2001). On attentional control as a source of residual shift costs: Evidence from twocomponent task shifts. Journal of Experimental Psychology: Learning, Memory, \& Cognition, 27, 640-653.

Jer sil d, A. T. (1927). Mental set and shift. Archives of Psychology (Whole No. 89).

$\mathrm{Kl}$ ein sorge, T. (2000). Explorations in the structure of task spaces. Psychologica Belgica, 40, 247-257.

Kl ein sor ge, T., \& Heu er, H. (1999). Hierarchical switching in a multi-dimensional task space. Psychological Research, 62, 300-312.

$\mathrm{Kl}$ ein sorge, T., Heu er , H., \& Schmidt ke, V. (2001). Task-set reconfiguration with binary and three-valued task dimensions. Psychological Research, 65, 192-201.

Loft u s, G. R., \& Masson, M. E. J. (1994). Using confidence intervals in within-subject designs. Psychonomic Bulletin \& Review, 1, 476-490.

Logan, G. D. (1985). Executive control of thought and action. Acta Psychologica, 60, 193-210.

Logan, G. D., \& Bundesen, C. (2003). Clever homunculus: Is there an endogenous act of control in the explicit task-cuing procedure? Journal of Experimental Psychology: Human Perception \& Performance, 29, 575-599.

Logan, G. D., \& Gordon, R. D. (2001). Executive control of visual attention in dual-task situations. Psychological Review, 108, 393-434. 
Logan, G. D., \& Schul kind, M. D. (2000). Parallel memory retrieval in dual-task situations: I. Semantic memory. Journal of Experimental Psychology: Human Perception \& Performance, 26, 1072-1090.

Mayr, U., \& Kl ieg1, R. (2000). Task-set switching and long-term memory retrieval. Journal of Experimental Psychology: Learning, Memory, \& Cognition, 26, 1124-1140.

McLeod, P. (1977). A dual task response modality effect: Support for multiprocessor models of attention. Quarterly Journal of Experimental Psychology, 29, 651-667.

Medin, D. L., Gol dst one, R. L., \& Gent ner, D. (1993). Respects for similarity. Psychological Review, 100, 254-278.

Meiran, N. (1996). Reconfiguration of processing mode prior to task performance. Journal of Experimental Psychology: Learning, Memory, \& Cognition, 22, 1423-1442.

Meir an, N. (2000a). Modeling cognitive control in task-switching. Psychological Research, 63, 234-249.

Meir an, N. (2000b). Reconfiguration of stimulus task sets and response task sets during task switching. In S. Monsell \& J. Driver (Eds.), Attention and performance XVIII: Control of cognitive processes (pp. 377-399). Cambridge, MA: MIT Press.

Meir a n, N., Chor ev, Z., \& Sa pir, A. (2000). Component processes in task switching. Cognitive Psychology, 41, 211-253.

Meiran, N., \& Marciano, H. (2002). Limitations in advance task preparation: Switching the relevant stimulus dimension in speeded same-different comparisons. Memory \& Cognition, 30, 540-550.

Meut er, R. F. I., \& Al 1 port , A. (1999). Bilingual language switching in naming: Asymmetrical costs of language selection. Journal of Memory \& Language, 40, 25-40.

Monsel 1, S., Yeung, N., \& Azuma, R. (2000). Reconfiguration of task-set: Is it easier to switch to the weaker task? Psychological Research, 63, 250-264.

Nor man, D. A., \& Shall ice, T. (1986). Attention to action: Willed and automatic control of behavior. In R. J. Davidson, G. E. Schwartz, \& D. Shapiro (Eds.), Consciousness and self-regulation: Advances in research and theory (Vol. 4, pp. 1-18). New York: Plenum.

Pashl er, H. \{E.\} (1990). Do response modality effects support multiprocessor models of divided attention? Journal of Experimental Psychology: Human Perception \& Performance, 16, 826-842.

Pashl er, H. E. (1998). The psychology of attention. Cambridge, MA: MIT Press.
Pashl er, H. E. (2000). Task switching and multitask performance. In S. Monsell \& J. Driver (Eds.), Attention and performance XVIII: Control of cognitive processes (pp. 278-307). Cambridge, MA: MIT Press.

Posner, M. I., \& Sn yder, C. R. R. (1975). Attention and cognitive control. In R. L. Solo (Ed.), Information processing and cognition: The Loyola Symposium (pp. 55-85). Hillsdale, NJ: Erlbaum.

Psychol ogy Soft ware Tool s, Inc. (2000). E-prime (Version 1.0). Pittsburgh, PA: Author.

Roger s, R. D., \& Mon sel 1, S. (1995). Costs of a predictable switch between simple cognitive tasks. Journal of Experimental Psychology: General, 124, 207-231.

Rubinst ein, J. S., Meyer, D. E., \& Evan s, J. E. (2001). Executive control of cognitive processes in task switching. Journal of Experimental Psychology: Human Perception \& Performance, 27, 763-797.

Sohn, M.-H., \& Ander son, J. R. (2001). Task preparation and task repetition: Two-component model of task switching. Journal of Experimental Psychology: General, 130, 764-778.

Sohn, M.-H., \& Carl son, R. A. (2000). Effects of repetition and foreknowledge in task-set reconfiguration. Journal of Experimental Psychology: Learning, Memory, \& Cognition, 26, 1445-1460.

Spect or, A., \& Bieder man, I. (1976). Mental set and shift revisited. American Journal of Psychology, 89, 669-679.

Sudevan, P., \& Tayl or, D. A. (1987). The cuing and priming of cognitive operations. Journal of Experimental Psychology: Human Perception \& Performance, 13, 89-103.

Tver sky, A. (1977). Features of similarity. Psychological Review, 84, 327-352.

\section{NOTE}

1. Within the task switching literature, the term task set is alternatively used to describe a state of preparedness for a task and a group of component operations necessary for performing a task. The second definition is used throughout this article. Thus, task set refers to the mental representations and processes associated with a task.

(Manuscript received January 26, 2003; accepted for publication March 24, 2003.) 